\section{Curling with kinesin}

Curling of a sheet of cells is a common morphogenetic process used at several developmental stages, including gastrulation and neurulation. A simplified, analogous process occurs during development of Volvox carteri, a multicellular green alga in which a spherical monolayer of cells must turn itself inside out. Recent work by Kirk and colleagues (Ce/l 113, 743-753 (2003)) has identified a new kinesin microtubule motor that is necessary for generating the cell movements required for this inversion process.

At the time of inversion, the spherical monolayer of cells is interconnected by a band of cytoplasmic bridges. The monolayer is made of flask-shaped cells with their wide ends facing into the interior of the sphere and their narrow ends facing outwards. The plane of the cytoplasmic bridge network connects these cells near their wide ends. Curling is initiated by cells moving with respect to their cytoplasmic bridge such that they go from being connected at their wide ends to being connected at their narrow ends (see arrows in the left image). This movement forces the cell sheet to curl sharply outwards and back over itself.

Mutants in the inversion process were identified 30 years ago, but because inversion is necessary to acquire the adult configuration (that is, reproductive cells on the interior) inversionless mutants are unable to mate, and so normal Mendelian analysis could not be used to identify the genes responsible. Now, Kirk and colleagues use the Jordan temperature-sensitive transposon to generate transposontagged mutants, which can then be used to clone the genes involved. One of the inversion mutants identified, InvA, was found to have a defect in cell movements such that the cytoplasmic bridges remain at the wide end of the cells (see arrowheads in the image on the right), and, thus, the curling process does not occur. By sequence analogy, the identified gene was predicted to be a plus-end-directed kinesin microtubule motor. InvA is expressed at the time of inversion and the protein is localized to the cytoplasmic bridge.

Identification of a microtubule motor gave immediate insight into the mechanism of inversion, because a network of microtubules running the length of the flask-shaped cells and passing just adjacent to the cytoplasmic bridge were known to be important for the inversion process.
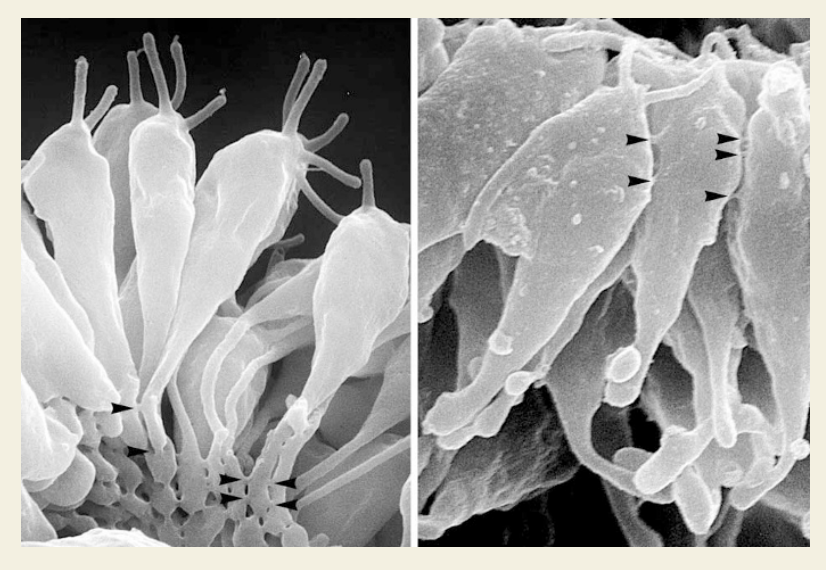

Flask cells of an inverting wild-type Volvox embryo (left) and an inversionless mutant (right). Arrowheads indicate cytoplasmic bridges that link neighbouring cells. In the wild-type embryo, the cells have moved so that they are linked by bridges at their narrow ends. However, the mutant cells have failed to move, so they remain linked near their wide ends. Figure adapted from Nishii et al. (c) (2003) with permission from Elsevier Science.

From these observations, the authors propose a model for how InvA might generate the necessary cell movements. Microtubules are aligned with their plus ends terminating at the narrow end of the cell. Thus, the plus end motor activity of InvA would move it towards the narrow end of the cell. However, if InvA is attached to the cytoplasmic bridge structure, as suggested by its localization, this motor movement would actually tend to move the microtubule network in the opposite direction. This could function to move the cell contents toward the wide end of the cell, leaving the cytoplasmic bridge connecting cells at their narrow tips - the correct confirmation to initiate inversion.

More work will be required to determine if this is indeed the mechanism. It will also be interesting to determine if analogous mechanisms are used in gastrulation or neurulation. The simplicity of Volvox inversion, combined with the new genetic tools for identifying mutant genes, should be fertile ground for identifying more of the molecular players in this prototypical morphogenetic event.

JACK HORNE observed for $\beta_{1}$ integrin knockouts ${ }^{8}$. This difference may be explained by redundancy between talin1 and talin2. Talin1-deficient cells have defects in spreading and focal adhesion formation; nonetheless differentiated talin1-null fibroblasts can form focal adhesions and spread, possibly through upregulation of talin2 expression ${ }^{8}$. The presence of talin2 in talin1-null fibroblasts complicates analysis of talin function. Nonetheless, loss of talin1, even in the presence of talin2, prevents formation of the 2-pN linkage and this effect is reversed by re-expression of talin1. Thus, either talin2 is unable to mediate the $2-\mathrm{pN}$ link, or it is not expressed at a sufficient level to do so in the early passage talin1-knockout cells. This question might be addressed by measuring breaking forces in talin1-knockout cells expressing increased levels of talin2.

The $\beta_{3}$ integrin specificity ${ }^{3}$ of the early 2 -pN force peak raises fascinating issues as to its potential biological significance. The fibronectin-coated beads can bind $\beta_{1}$ integrins (ref. 4), as well as $\beta_{3}$ integrins; however, $\alpha_{v} \beta_{3}$ inhibitors or knockout of integrin $\beta_{3}$ was sufficient to block the 2-pN force peak. Binding of fibronectin to $\beta_{1}$ integrins results in the formation of talin-containing adhesion complexes and mediates cell spreading, migration and matrix assembly. Furthermore, although $\beta_{3}$ and $\beta_{1}$ integrins differ in their recruitment of some focal adhesion proteins, they both bind talin ${ }^{9}$. The 2-pN force peak, however, is specific for $\beta_{3}$ integrin. The embryonic-lethal phenotype of mice lacking talin 1 (ref. 8 ) is more severe than that of $\beta_{3}$-integrin-deficient mice, which are viable and fertile $\mathrm{e}^{10}$. This indicates that talin has other important roles in addition to its interaction with $\beta_{3}$ integrin and is consistent with talin having important interactions with other integrins. Nonetheless, although it is probable that talin mediates links between many integrins and actin, it is important to clarify the functional significance of the $\beta_{3}$-specific 2 -pN force peak.

For the initial talin-mediated actin tethers to mature into larger adhesion complexes, additional proteins must be recruited and the ECM-actin links reinforced. As well as providing the initial tethers, talin may contribute to reinforcement by generating a positive feedback loop, resulting in biosynthesis of phosphatidylinositol-4,5-bisphosphate (PtdIns: $\mathrm{P}_{2}$ ), a lipid regulator of cytoskeletal 